\title{
JUSTICIA SOCIAL, UNA ALTERNATIVA DE LA JUSTICIA PENAL PARA LAS NIÑAS, NIÑOS Y ADOLESCENTES
}

\author{
Social justice, criminal justice's alternative for children and adolescents
}

Margarita CRUZ TORRES

\begin{abstract}
Sumario:
I. Introducción: Una mirada teórica de la justicia social de los derechos de las niñas, niños y adolescentes. II. Reconocimiento jurídico-penal, una limitante para el desarrollo de la justicia social. III. Limitaciones del principio rector "interés superior del niño". IV. Justicia y derechos fundamentales. V. Principio de igualdad para la infancia y la adolescencia. VI. Derechos más allá de la justicia. VII. Consideraciones finales.
\end{abstract}

Resumen. El trabajo tiene como objetivo analizar la justicia para las niñas, niños y adolescentes en el ámbito de sus derechos desde una perspectiva teórica, demostrando que existe una interpretación errónea respecto de la misma. Los principales debates en torno al tema de los derechos de la infancia y la adolescencia, se plantean a partir de la falta de una teoría que permita reconocerlos como verdaderos titulares de derechos y no solo como objeto de protección. Las niñas, niños y adolescentes tienen derecho a vivir en condiciones que garanticen su pleno desarrollo, lo cual implica hacer el señalamiento de que la justicia social para la infancia y la adolescencia, es una asignatura pendiente en el Estado Democrático de Derecho.

Palabras clave: Derecho de la infancia, derechos fundamentales, justicia penal, justicia social, Estado.

Abstract. This work examines the criminal justice within the scope of their rights from a theoretical perspective, nothing that there is a misunderstanding regarding the same. It affirm that the main debates around the issue of the rights of childhood and adolescence arises from the lack of a theory that children can recognize as true guardians of rights and not just as objects of protection. It affirm that children and adolescents have the right to live in conditions that ensure their full development, which involves making de claim that adolescents justice remains an unsettled issue in the Democratic State.

Key words: Children rights, fundamental rights, criminal justice, social justice, State.

\footnotetext{
${ }^{1}$ Maestra en Derecho Penal, profesora de Teoría del Delito, la Pena y la Restauración en la Licenciatura de Criminología de la Facultad de Derecho Universidad Autónoma de Querétaro, docente certificado en temas del "Nuevo Sistema Procesal Penal" por la SETEC, Diplomado en Derechos Humanos, Género y Justicia (INACIPE), Diplomado en Prevención del Delito con Participación Ciudadana (UAQ), Diplomado en Justicia, Administración y Delincuencia Organizada (IAPQ), Miembro de la Academia Mexicana de Criminología y Licenciada en Derecho por la Universidad Autónoma de Querétaro (UAQ).
} 
I. INTRODUCCIÓN: UNA MIRADA TEÓRICA DE LA JUSTICIA SOCIAL DE LOS DERECHOS DE LAS NIÑAS, NIÑOS Y ADOLESCENTES

A partir de la Convención de los Derechos del Niño se integró a las personas menores de 18 años a la comunidad jurídica, otorgando a las niñas, niños y adolescentes, la condición de sujetos de derechos y deberes, lo cual les había sido negado durante toda la modernidad.

El establecimiento de un sistema integral de justicia para adolescentes que hoy consagra la Carta Magna, introduce la idea de responsabilidad penal limitada en la población adolescente, además del reconocimiento de las garantías procesales ante la actividad punitiva del Estado, lo cual resulta un eufemismo, al identificarse como un sistema represivo opuesto al interés superior del niño, debido a que para hablar de responsabilidad, es menester reconocer la libertad y por ende la igualdad.

El llamado reconocimiento a los derechos fundamentales de las niñas, niños y adolescentes se limita sólo a la justicia penal con la aplicación de las garantías procesales, quedando sin abordar derechos como el acceso a la educación, la salud, a una vida en familia, entre otros. Se requiere de una teoría de los derechos de la niñez y la adolescencia en la se identifique la verdadera esencia de la justicia.

La justicia de niñas, niños y adolescentes es una asignatura pendiente en el Estado Democrático de Derecho, la cual se encuentra limitada a la administración de justicia penal. La justicia para la infancia y la adolescencia debería ser entendida como la justicia social que lleve a lograr el pleno ejercicio de sus derechos fundamentales.

II. RECONOCIMIENTO JURÍDICO-PENAL, UNA LIMITANTE PARA EL DESARROLLO DE LA JUSTICIA SOCIAL

Uno de los principales debates en torno al tema de los derechos de la infancia y la adolescencia, se plantea a partir de la falta de una teoría que permita reconocer a las niñas, niños y adolescentes como verdaderos titulares de derechos y no sólo como objeto de protección.

No obstante el reconocimiento formal de la titularidad de los derechos de los niños, niñas y adolescentes presenta dificultades en su aplicación. La premisa implícita es siempre la visión de la infancia y la adolescencia como seres incapaces y dependientes, por lo que quedan a la voluntad de los padres el ejercicio de la patria potestad y del Estado garantizar el ejercicio de su autonomía, anulada bajo la supuesta protección integral, reducida al otorgamiento de garantías procesales y las diversas interpretaciones en torno al principio del interés superior del niño.

Afirmar que las niñas, niños y adolescentes tienen derecho a vivir en condiciones que garanticen su pleno desarrollo, implica hacer el señalamiento de que la justicia para ellos, es una asignatura pendiente en el Estado Democrático de Derecho. El Estado tiene una enorme deuda con la infancia y la adolescencia en aspectos sustantivos de justicia social.

Los principios de la justicia social determinan la división de ventajas que permiten suscribir un convenio sobre las participaciones distributivas correctas, que proporcionan un 
modo de asignar derechos y deberes en las instituciones básicas de la sociedad y definen la distribución apropiada de los beneficios y las cargas de la cooperación social. ${ }^{2}$

Formular un debate profundo del tratamiento jurídico de las niñas, niños y adolescentes, implica reconocer en primer término el vacío que existe en el concepto de "derechos" durante la minoría de edad de las personas.

La Convención sobre los Derechos del Niño es el referente fundamental en el reconocimiento jurídico de la infancia y la adolescencia, considerando que "la libertad, la justicia y la paz en el mundo se basan en el reconocimiento de la dignidad intrínseca de los derechos iguales e inalienables de todos los miembros de la familia humana." 3 Sin embargo, el Estado se resiste a determinar los alcances de cada uno de sus derechos, es importante reconocer que las niñas, los niños y los adolescentes tienen derecho a cuidados y asistencia especiales.

\section{LIMITACIONES DEL PRINCIPIO RECTOR “INTERÉS SUPERIOR DEL NIÑO”}

La aplicación del principio del interés superior del niño como la consideración primordial que debe atender el Estado en su intervención, es interpretada en cualquier sentido como criterio rector, el cual es utilizado desde diversas posturas ideológicas. Dicha interpretación ha servido para encubrir la necesidad de una discusión de fondo sobre los problemas relacionados con la exigibilidad de derechos que lleven a determinar claramente sobre los contenidos, alcances y mecanismos de protección.

Este principio se cita indistintamente en temas de carácter jurídico, social y hasta político, para justificar la acción del Estado en sus diversos ámbitos, que lejos de favorecer a los titulares de derechos, ha provocado un desacierto en su aplicación, pues pretender una falsa claridad ignora la necesidad de ser subsanada.

El principio del interés superior del niño ha sido desvirtuado para introducir nuevamente el paternalismo discrecional, no sólo en las decisiones judiciales, sino con ello se justifica la ausencia de políticas públicas al respecto. Este principio se llega a utilizar para justificar la violencia de Estado en contra de las niñas, los niños y los adolescentes. La falta de límites del mismo, hace vulnerables los intereses jurídicamente protegidos, los convierte en inaplicables al no haber claridad respecto de las obligaciones del Estado, pues resultan ignorados los deberes con la infancia y la adolescencia.

\section{JUSTICIA Y DERECHOS FUNDAMENTALES}

No se puede concebir la justicia para niñas, niños y adolescentes desde el enfoque teórico, sin abordar el tema de los derechos fundamentales, que de acuerdo a la propuesta de Ferrajoli son "aquellos derechos subjetivos que las normas de un determinado ordenamiento jurí-

\footnotetext{
${ }^{2}$ RAWLS, John, Teoría de la Justicia, $2^{\text {a }}$ edición, traducción de María Dolores González, México, Fondo de Cultura Económica, 1993, p. 21.

${ }^{3}$ Cfr. Convención sobre los Derechos del Niño en: http://www.sre.gob.mx/derechoshumanos/docs/28.pdf. 14 de agosto de 2014 .
} 
dico atribuyen universalmente a todos en tanto personas, ciudadanos y/o personas capaces de obrar". ${ }^{4}$

Todo Estado que se proclame garantista se debe sustentar en la aplicación de los derechos fundamentales de las personas, en la dignidad y el valor de la persona humana en un ámbito de libertad.

Actuar libremente según Kant, es actuar autónomamente. Y actuar autónomamente es actuar conforme a una ley que me doy a mí mismo, no conforme con dictados de la naturaleza o de la convención social. ${ }^{5}$

Los derechos fundamentales corresponden a aquellas facultades o expectativas de todos, que definen las connotaciones sustanciales de la democracia y que están constitucionalmente sustraídas al arbitrio de las mayorías como límites o vínculos insalvables de las decisiones del gobierno; tales como el derecho a la vida, libertad, la subsistencia, la salud, la instrucción, la conservación del ambiente y otros similares. ${ }^{6}$

El garantismo como modelo alternativo de Estado de Derecho, destaca las insuficiencias del modelo liberal, ${ }^{7}$ como una respuesta inconclusa que es utilizada eufemísticamente tanto en los discursos políticos como en la exposición de motivos de iniciativas de reformas legales que aluden a la justicia de niñas, niños y adolescentes, lo que resulta necesario es expresar de manera clara las obligaciones del Estado frente a la infancia y la adolescencia, garantizar su pleno ejercicio, así como la obligación positiva de generar las medidas necesarias para asegurar que no sean violentados sus derechos fundamentales.

En la doctrina liberal, Estado de Derecho no sólo significa subordinación de los poderes públicos a las leyes generales del país, que es un límite solo formal, sino también subordinación de las leyes al límite material del reconocimiento de los derechos fundamentales considerados constitucionalmente y por tanto, en principio "inviolables".

Adam Smith señala que "en la ética se expresa lo que el hombre piensa de sí mismo y lo que el hombre espera del hombre". ${ }^{9}$ El derecho de igualdad dentro de la categoría de niñas, niños y adolescentes, implica el derecho a que no se encuentren privados de crear y desarrollar un proyecto de vida que es sustancial para procurar sentido a la existencia y requiere para su desarrollo condiciones de una vida digna, de seguridad e integridad para las personas. Derechos fundamentales que se identifican como valores superiores que permiten generar calidad de vida, a la niñez y la adolescencia.

Una sociedad es homogénea, en términos de Garzón Valdés, cuando todos sus miembros tienen la posibilidad de ejercer los derechos vinculados con la satisfacción de sus bienes básicos o primarios, éstos son necesarios para la realización de todo plan de vida y aunado con los derechos que se encuentran vinculados conforman lo que este autor llama "coto vedado". ${ }^{10}$

Si el objeto de los derechos fundamentales hace referencia al ámbito de la libertad, que implica garantizar el tratamiento normativo en que aquellos consisten, no se puede negar que

\footnotetext{
${ }^{4}$ FERRAJOLI, Luigi, Los Fundamentos de los Derechos Fundamentales, Madrid, Trotta, 2005, p. 290.

5 KANT, Immanuel, Fundamentación de la Metafísica de las Costumbres, Traducción por Manuel García Motente, Porrúa, México, 2010, p. 122.

6 FERRAJOLI, Luigi, Derecho y Razón. Teoría del Garantismo Penal, $4^{\circ}$ edición, Madrid, Editorial Trotta, 200, p. 883 .

7 Op.cit, nota 3, p. 290.

8 BOBBIO, Norberto, Liberalismo y Democracia, México, Fondo de Cultura Económica, 20o6, p. 19.

9 SMITH, Adam, Teoría de lo Sentimientos Morales, México, Fondo de Cultura Económica, 1983, p. 19.

${ }^{10}$ GARZÓN VALDÉS, Ernesto, "Mercado y Justicia”, Revista Isonomía, ITAM/ Fontamara, 2, 1995, p. 23, cit. por VÁZQUEZ, Rodolfo, Entre la Libertad y la Igualdad, Introducción a la Filosofía del Derecho, Madrid, 
éste es el mismo durante la minoría y la mayoría de edad del individuo y lo único que varía son las circunstancias personales en la que éste se encuentra. Por ello, el derecho a la vida y la integridad física, la libertad ideológica y de conciencia, la libertad personal, la intimidad y la propia imagen, la libertad de reunión y manifestación, la libertad de circulación y residencia, la libertad de expresión y creación artística, la libertad de asociación, la tutela judicial efectiva o la educación, por citar algunos de ellos, constituyen los ámbitos de libertad a través de los cuales el individuo se autodetermina y se autorepresenta. ${ }^{11}$

En la doctrina de los derechos de las personas se sustenta que todos, indistintamente tienen por naturaleza derechos fundamentales, como el derecho a la vida, a la libertad, a la seguridad, a la felicidad, que el Estado o más concretamente, aquellos que en un determinado momento histórico detentan el poder legítimo de ejercer la fuerza para obtener obediencia a sus mandatos, deben respetar no invadiéndolos y garantizarlos frente a cualquier intervención posible por parte de los demás. ${ }^{12}$

Los derechos fundamentales solo existen en cuanto alcanzan el carácter de ejecutivo, en el momento en que son operativamente positivados, de modo tal que su reconocimiento no quede al arbitrio de un órgano cualquiera, ante el cual pudiera plantearse un cuestionamiento en torno a estos derechos. El fundamento último de tales derechos, así como el derecho positivo en su conjunto, radica en su asunción como tema relevante en el aspecto deliberativo. La existencia de los derechos fundamentales como se expone en el efectivo tránsito de los mismos, desde la discusión y aprobación en el momento político, hasta su formalización al Estado ejecutivo, es decir, su positividad jurídica y aplicación. ${ }^{13}$

El derecho de la infancia y la adolescencia al sustentarse en principios que deben ser reconocidos como normas que es menester observar, no sólo porque encuentren posible una condición de su regulación en un texto legal, sino por ser un imperativo de justicia basado en necesidades y capacidades humanas. La divergencia sustancial entre el ser legal del derecho y el deber ser constitucional determinaría, según Ferrajoli ${ }^{14}$ la frecuente existencia de normas formalmente válidas o vigentes, pero sustancialmente inválidas o inconstitucionales.

Como lo he mencionado, no existe claridad en la aplicación del principio denominado “interés superior del niño", como uno de los principios del discurso garantista, lo cual genera la aplicación de políticas públicas inadecuadas.

El interés superior del niño es un concepto interpretado por la Corte Interamericana de los Derechos Humanos de la siguiente manera: "la expresión interés superior del niño... implica que el desarrollo de éste y el ejercicio pleno de sus derechos deben de ser considerados como criterios rectores para la elaboración de normas y la aplicación de éstas en todos los órdenes relativos a la vida del niño". 15

Trotta, 2010, p. 266.

${ }^{11}$ ALAÉZ CORRAL, Benito, Minoría de Edad y Derechos Fundamentales, Madrid, Tecnos, 2003, pp. 173-174 en ISLAS DE GONZÁLEZ, Mariscal y CARBONELL, Miguel, Constitución y Justicia para Adolescentes, México, UNAM, 2007, p. 3.

${ }^{12}$ BOBBIO, Norberto, op. cit, nota 7, p. 11.

13 PICCATO, Antonio, "Algunas Aportaciones de Hans Kelsen al Constitucionalismo Contemporáneo", Revista Ciencia Jurídica y Constitución Ensayos e Homenaje a Rolando Tamayo y Salmorán, Fernando Serrano Migañón y otros, México, Porrúa-UNAM, 2008, p. 405.

14 FERRAJOLI, Luigi, El Garantismo y la Filosofía del Derecho, traducción de Gerardo Pisarello y otros, Colombia, Universidad del Externado de Colombia, 200o, p. 14.

${ }^{15}$ Criterios de la Corte Interamericana de Derechos Humanos, www.derechoshumanos.gob. mx/criterios/.../ menores deedad.htm. 14 de agosto de 2014. 


\section{PRINCIPIO DE IGUALDAD PARA LA INFANCIA Y LA ADOLESCENCIA}

El principio de igualdad, en el que se sintetiza la idea de justicia formal en el sentido tradicional de la palabra, dice pura y simplemente que deben ser tratados de igual modo todos aquellos que pertenezcan a la misma categoría, pero los criterios para establecer las categorías los vamos a encontrar en los llamados criterios o principios de justicia, como "a cada uno según su mérito", "a cada uno según su necesidad”, "a cada uno según su rango", etc. Son conformes al principio de igualdad tanto la máxima "a cada uno la misma cosa", si en base al criterio adoptado resulta que todos los seres humanos son iguales, cuanto la máxima "a cada uno una cosa diferente", si en base al criterio adoptado resulta que todos los seres humanos son distintos. También el privilegio puede ser considerado en último término como una aplicación del principio de igualdad, cuando se logra demostrar que aquel individuo tiene tales características singulares respecto a la disciplina en cuestión como para constituir una categoría por sí mismo. ${ }^{16}$

Esto permite exigir los mismos derechos para la niñez y la adolescencia que para los adultos, lo cual implica crear mecanismos de garantía como una tarea de la política criminal y un giro al abordaje de la protección de los derechos de la infancia y la adolescencia, como el de la Doctrina de la Protección Integral, entendida como la obligación del Estado de generar las condiciones para el pleno ejercicio de los derechos fundamentales de las niñas, niños y adolescentes, sobre el reconocimiento de la capacidad de ellos mismos para exigir su cumplimiento.

Uno de los intentos por construir una teoría de los derechos de la infancia y la adolescencia, es la Doctrina de la Protección Integral planteada por Emilio García Méndez, ${ }^{17}$ la cual se identifica como: "el conjunto de instrumentos jurídicos de carácter internacional que expresan un salto cualitativo fundamental en la consideración social de la infancia”. Reconociendo como antecedente directo la Declaración de los Derechos del Niño, esta doctrina condensa la existencia de cuatro instrumentos básicos: La Convención Internacional sobre los Derechos del Niño, Las Reglas Mínimas de las Naciones Unidas para la Administración de la Justicia Juvenil (Reglas de Beijín), Las Reglas Mínimas de las Naciones Unidas para los Jóvenes Privados de Libertad y Las Directrices de las Naciones Unidas para la Administración de la Justicia Juvenil (Reglas de RIAD).

Para la Corte Interamericana de los Derechos Humanos, en su opinión Consultiva $17 / 2002,{ }^{18}$ sobre la condición jurídica y derechos humanos de la infancia y la adolescencia, los deberes del Estado en relación con los niños, niñas y adolescentes no se agotan en actos de abstención por parte de los poderes públicos, sino que requieren y exigen de políticas activas para la preservación de sus derechos. Por lo que al referirse a los deberes del Estado, señala como antecedentes que la vigencia de los derechos y garantías reconocidas en la Convención Americana, no es plena respecto a los niños, niñas y adolescentes como sujetos y autores en jurisdicción, por asumirse que la obligación de protección del Estado es para

\footnotetext{
${ }^{16}$ BOBBIO, Norberto, El Tiempo de los Derechos, Madrid, Sistema, 1991, p.45.

${ }^{17}$ GARCÍA MÉNDEZ, Emilio, Infancia y Adolescencia, De los Derechos y De la Justicia, $3^{\text {a }}$ edición, México, Fontamara, 2007, p. 29.

${ }^{18}$ Opinión Consultiva 17/2002, sobre la condición jurídica y derechos humanos del niño, http://www.iin/pdf/ publicaciones/Corte_in. 15 de agosto de 2014.
} 
suplir la falta de plenitud de juicio de la niñez y la adolescencia, puede hacer pasar a segundo plano dichas garantías. Por lo que impone al Estado una serie de obligaciones positivas de protección, tales como la obligación de respetar los derechos y libertades reconocidos en ella y organizar el poder público para garantizar a las personas bajo su jurisdicción, el libre y pleno ejercicio de los Derechos Humanos. Dicha obligación general impone a los Estados que forman parte, el deber de garantizar el ejercicio y disfrute de los derechos de los individuos en relación con el poder del Estado y también en relación con actuaciones de terceros particulares; en este sentido, los Estados parte de la Convención Americana, tiene el deber de tomar las medidas positivas que aseguren la protección a los niños, niñas y adolescentes contra las diferentes formas de violencia. En sus relaciones con las autoridades públicas, se desprenden de las normas contenidas en la Convención sobre los Derechos del Niño, la limitación del poder del Estado en el ámbito de la vida personal y familiar de las niñas, niños y adolescentes, pero al mismo tiempo, la obligación del Estado de que, sin necesidad de la exigencia de un derecho, hacer vigente el mismo, por el hecho de tratarse de derechos fundamentales del individuo. Esto requiere la adopción de medidas, entre otras, de carácter económico, social y cultural, como lo es el derecho a la educación que gradualmente supera la vulnerabilidad de las niñas, niños y adolescentes. El Estado como responsable del bien común, debe en igual sentido, resguardar el rol preponderante de la familia en la protección de la niñez y adolescencia y prestar asistencia del poder público para la implementación de medidas que favorezcan el adecuado desarrollo de las niñas, niños y adolescentes.

Para Rawls "una concepción general de la justicia puede ser expresada como todos los valores sociales -libertad y oportunidad, ingresos y riqueza, así como las bases del respecto a sí mismo- habrán de ser distribuidos igualitariamente a menos que una distribución desigual de alguno o de todos estos valores redunde en una ventaja para todos. La injusticia consistirá en las desigualdades que no benefician a todos." ${ }^{19}$

La exclusión de la niñez y la adolescencia del sistema penal, justificada en el discurso tutelar por la necesaria aflictividad de este último, ${ }^{20}$ significó la cancelación de las garantías constitucionales que son base de legitimación de la intervención penal, lo cual implica que al amparo de un discurso paternalista sustentado en la debilidad de la infancia y la adolescencia, no canceló la posibilidad que el sistema penal otorga a quién es imputado por haber cometido un delito, de garantizar su defensa y protegerlo de las posibilidades de abuso que el propio sistema comporta. ${ }^{21}$

El problema se presenta ante la necesidad de entender que los vulnerables no son las niñas, niños y adolescentes, sino sus derechos, por esa razón lo que hay que proteger son sus derechos; ya que protegiendo a una persona, paradójicamente, se corre el riesgo de ponerla en peligro y de cancelar sus posibilidades para que ella misma, como sujeto libre y autónomo, sea capaz de aprender de su propia experiencia. ${ }^{22}$

$\mathrm{Al}$ proteger sus derechos, en cambio, nunca se presentará el riesgo de dañarlos y, en ese sentido, se le estará dejando en libertad para que sea capaz de construir su sentido de res-

\footnotetext{
19 Op. cit, nota 1, p. 69.

${ }^{20}$ Por la naturaleza violenta de la prisión, pero fundamentalmente, por considerar que las niñas, niños y adolescentes no tienen capacidad para comprender la naturaleza del delito ni para asimilar el contenido pedagógico y resocializante de la pena.

${ }^{21}$ GONZÁlEZ PLACENCIA, Luis, La Política Criminal en Materia de Niñas, Niños y Adolescentes en Conflicto con la Ley Penal en México, Programa de Cooperación Unión Europea-México, Instituto Nacional de Ciencias Penales, México, 2006, p. 14.

${ }^{22}$ Ibid., p. 143 .
} 
ponsabilidad frente al otro. Por lo que la alimentación, salud, educación, recreación, trabajo o juego, son elementos que contribuyen al desarrollo pleno de la personalidad en un medio ambiente sano, constituyen asignaturas que, como derechos fundamentales de la infancia y la adolescencia, requieren ser plenamente satisfechos, incluso en las situaciones en la que se llegue a la privación de la libertad.

\section{DERECHOS MÁS ALLÁ DE LA JUSTICIA}

De lo anterior se desprende que la diferencia, por tanto, entre un sistema penal puramente retributivo y un sistema penal de garantías, radica, precisamente en el reconocimiento de los derechos como límite a cualquier forma de intervención del Estado frente al destinatario de tales derechos.

Señala Manuel Atienza ${ }^{23}$ que la justicia viene a ser como el valor jurídico por antonomasia, sin embargo no es el único, pero puede considerarse como un valor de totalidad, de manera que afirmar que un determinado derecho es justo viene a ser una forma sintética, es decir, que satisface una serie de valores como la igualdad, la libertad y la seguridad.

La idea de justicia, siempre nos ofrecerá una diversidad de concepciones, hoy buscamos aquella que como pieza del derecho no sólo se identifique con la realidad social, sino que responda a los retos que el garantismo nos impone: limitar el poder del Estado a través de la estricta legalidad.

El objetivo primario de los principios de justicia social es la estructura básica de la sociedad, la disposición de las instituciones sociales más importantes en un esquema de cooperación. Principios que habrán de gobernar la asignación de derechos y deberes en estas instituciones y que habrán de determinar la correcta distribución de los beneficios y las cargas de la vida social. ${ }^{24}$

Se manifiesta así uno de los puntos críticos en la materia, ya que existe un vacío entre los estudiosos de los derechos de las niñas, niños y adolescentes, que no logran insertar desde la teoría la eficacia a la norma jurídica.

Hay una crisis profunda y creciente del derecho, que se manifiesta de diversas formas. Un aspecto de ellas es la crisis de legalidad, del valor vinculante asociado a las reglas por los titulares de los poderes públicos. Se expresa por la ausencia y la ineficiencia de los controles y por, tanto, en la "variada y llamativa fenomenología de la ilegalidad del poder". ${ }^{25}$

Se requiere crear los mecanismos que garanticen la efectividad de los derechos de la infancia y la adolescencia y un cambio radical en la forma de abordar esos derechos desde la perspectiva del Estado Democrático de Derecho, los cuales deben tener como premisa fundamentalmente la defensa del individuo de los abusos del poder.

$\mathrm{Al}$ referirse a los límites del poder del Estado, Norberto Bobbio ${ }^{26}$ señala que no siempre se distingue con precisión los límites del poder y las funciones del Estado, ambos tratados por el Estado Liberal. El liberalismo es una doctrina del Estado limitado, tanto con respecto

\footnotetext{
${ }^{23}$ ATIENZA, Manuel, El Sentido del Derecho, España, Ariel, 2001, p. 177.

${ }^{24}$ RAWLS, John, nota 1, op. cit. p. 62.

${ }^{25}$ FERRAJOLI, Luigi, Derecho y Garantías, La Ley del Más Débil, $2^{\text {a }}$ edición, Trotta, Madrid, 2001, p. 15.

${ }^{26}$ BOBBIO, Norberto, op. cit. nota 7, p. 17.
} 
a los poderes como a sus funciones. La noción común que sirve para representar al primero, es el Estado de Derecho y para representar el segundo, es el Estado Mínimo.

Hay condiciones significativamente preocupantes que demandan una atención no penal, tales como la disfunción familiar, el abandono escolar, adicciones, prostitución infantil, niños en situación de calle, falta de oportunidades de educación y desarrollo laboral, recreativas y culturales, pérdida de valores.

De lo anterior se deriva que "la igualdad es una compleja relación de personas regulada por los bienes que hacemos, compartimos e intercambiamos entre nosotros; no es una identidad de posesiones. Requiere entonces de una diversidad de criterios distributivos que reflejan la diversidad de los bienes sociales". ${ }^{27}$

La justicia para niñas, niños y adolescentes no se limita a administrar justicia penal, sino que debe ser entendida como el conjunto de derechos de los cuales son titulares, como la satisfacción de sus necesidades de alimentación, salud, educación y sano esparcimiento, entre otros, para lograr un desarrollo integral; como aquella que debe administrar el Estado, incluso antes de que el menor entre en conflicto con la ley penal, por ser un imperativo del mismo, proveer lo necesario para lograr el respeto a la dignidad de la niñez y la adolescencia y el ejercicio pleno de sus derechos.

De acuerdo a las Reglas de Bejín, la Justicia de Menores se ha de concebir como parte integrante del proceso de desarrollo nacional de cada país y deberá administrarse en el marco general de justicia social para todos los niños, niñas y adolescentes, de manera que contribuya a la protección de los jóvenes y al mantenimiento del orden pacífico de la sociedad. ${ }^{28}$

Para poder prevenir eficazmente la delincuencia es necesario que toda sociedad procure el desarrollo armónico de las niñas, niños y adolescentes, se construya y respete su personalidad, a partir de la primera infancia. Las políticas públicas y las medidas de esa índole deben incluir la creación de oportunidades, en particular educativas, formulación de doctrinas, criterios especializados para la prevención de la delincuencia, una intervención oficial que se guíe por la justicia y la equidad, cuya finalidad primordial sea velar por el interés general de las niñas, niños y adolescentes, la protección de su bienestar y desarrollo. ${ }^{29}$

La protección integral debe ser entendida como la minimización del derecho penal y la correlativa maximización de los derechos fundamentales, esta debe ser la premisa.

Una característica estructural y empírica de los estados constitucionales de derecho, es la virtual ilegitimidad del derecho vigente, causa de la divergencia entre la normatividad y la efectividad, entre el deber ser y ser del derecho, en tanto que el mismo se encuentra sometido al derecho. Por consiguiente, la dimensión sustancial y no sólo formal de la validez de las normas, depende ya no sólo de la forma en la cual son producidas, sino también de su contenido. ${ }^{30}$

Las normas son significados asociados a documentos normativos, como tales son definidas en el plano teórico e interpretadas en el plano dogmático y judicial. Pero ellas mismas, a

\footnotetext{
${ }^{27}$ WALZER, M, Las Esferas de la Justicia, Una defensa del pluralismo y la igualdad, Fondo de Cultura Económica, México, 1993, p. 31.

${ }^{28}$ Principio 1.4 Reglas Mínimas de las Naciones Unidas para la Administración de Justicia de Menores, Resolución 40/43 de 28 de noviembre de 1985. http://www.cndh.org.mx.armonlegu3/archivos/Instr... 16 de agosto de 2014 .

${ }^{29}$ Directrices de las Naciones Unidas para la Prevención de la Delincuencia Juvenil (Reglas de RIAD), adoptada por la Asamblea General de la ONU, en su resolución 45/112 de fecha 14 de diciembre de 1990 http://www. iin.oea.org/cad-RIAD.pdf. 14 de agosto de 2014.

${ }^{30}$ FERRAJOLI, Luigi, El Garantismo y la Filosofía del Derecho, op. cit, p. 46.
} 
su vez, están sometidas al menos en el Estado Constitucional de Derecho, a normas, ya sean formales o sustanciales sobre su producción, esto requiere la conformidad de los actos que las producen y la coherencia de los significados en que coinciden con las normas superiores respecto de ellos, bajo la pena de inexistencia en el primer caso y de invalidez sustancial en el segundo. ${ }^{31}$

Rescatar la validez normativa implica reconocer que los derechos fundamentales como facultades o expectativas de todos, que definen las connotaciones sustanciales de la democracia y que están constitucionalmente sustraídas al arbitrio de las mayorías como límites o vínculos insalvables de las decisiones de gobierno, tales como el derecho a la educación, a la conservación del ambiente y otros similares. ${ }^{32}$

Ampliar los alcances de la obligación del Estado de adoptar medidas a favor de la niñez y la adolescencia, genera el reconocimiento de que las niñas, niños y adolescentes son titulares de derechos y no solo como objetos de protección. Lo que implica que esos derechos requieren no solo que el Estado se abstenga de interferir indebidamente en las relaciones privadas o familiares del niño, sino también que, según las circunstancias adopte providencias positivas para asegurar el ejercicio y disfrute pleno de los derechos.

\section{CONSIDERACIONES FINALES}

Finalmente se puede afirmar que es el Estado, quien tiene el compromiso de respetar el pleno ejercicio de los derechos de las niñas, niños y adolescentes, así como generar los mecanismos desde la norma jurídica para su aplicación, no obstante, no puedan por sí mismos exigir su cumplimiento. Esa es la deuda histórica del Estado con la infancia y la adolescencia a la que me refiero.

El Estado Democrático de Derecho todavía se encuentra a distancia de alcanzar ese ideal de justicia social para las niñas, niños y adolescentes, que le permita ubicarse en un modelo de Estado Garantista; para ello es necesario armonizar las ideas que permitan construir una teoría de los derechos de la infancia y la adolescencia, que nos lleve a la eficacia de la norma jurídica a través de un verdadero sistema integral de justicia para niños, niñas y adolescentes, que no pueda ser otro que la indiscutible obligación del Estado de respetar y generar las políticas públicas que hagan realidad el goce y disfrute de sus derechos fundamentales.

BIBLIOGRAFÍA

ATIENZA, Manuel, El Sentido del Derecho, Ariel, España, 2000.

BOBBIO, Norberto, El Tiempo de los Derechos, Editorial Sistema, Madrid, 1991. Liberalismo y Democracia, Fondo de Cultura Económica, México, 2006.

FERRAJOLI, Luigi, Los Fundamentos de los Derechos Fundamentales, Trotta, Madrid, 2005. , Derecho y Razón, Teoría del Garantismo Penal, Trotta, Madrid, 2000. , Derecho y Garantías, La Ley del Más Débil, Trotta, Madrid, 2001.

\footnotetext{
${ }^{31}$ Ibid., p. 47.

${ }^{32}$ FERRAJOLI, Luigi, Derecho y Razón, Teoría del Garantismo Penal, Op. Cit, p. 883. 
, El Garantismo y la Filosofía del Derecho, Universidad del Externado de Colombia, Colombia, 2001.

GARCÍA, Emilio, Infancia y Adolescencia, de los Derechos y de la Justicia, Fontamara, 2007.

GONZÁLEZ PlACENCIA, Luis, La Política Criminal en Materia de Niñas, Niños y Adolescentes en Conflicto con la Ley Penal en México, Programa de Cooperación Unión Europea-México, Instituto Nacional de Ciencias Penales, México, 2006.

ISLAS DE GONZÁLEZ, Mariscal Olga y CARBONELL, Miguel, Constitución y Justicia para Adolescentes, UNAM, México, 2007.

KANT, Immanuel, Fundamentación de la Metafísica de las Costumbres, Traducción por Manuel García Motente, Porrúa, México, 2010.

RAWLS, John, Teoría de la Justicia, Fondo de Cultura Económica, México, 2012.

SERRANO MIGAÑóN, Fernando y VÁZQUEZ, Rodolfo, Ciencia Jurídica y Constitución, Porrúa y Facultad de Derecho de la UNAM, México, 2008.

SMITH, Adam, Teoría de los Sentimientos Morales, Fondo de Cultura Económica, México, 1983.

VÁZQUEZ, Rodolfo, Entre la Libertad y la Igualdad, Introducción a la Filosofía del Derecho, Trotta, Madrid 2010.

WALZER, Michael, Las Esferas de la Justicia, Una defensa del pluralismo y la igualdad, Fondo de Cultura Económica, México, 1993.

FUENTES ELECTRÓNICAS

Convención sobre los derechos del niño, http://www.sre.gob.mx/derechoshumanos/docs/28. $p d f$

Criterios de la Corte Interamericana de Derechos Humanos. http://www.derechoshumanos. gob.mx/criterios/.../menoresdeedad.htm

Opinión Consultiva 17/2002, sobre la Condición Jurídica y Derechos Humanos del Niño. http://www.iinoea.org/iin/pdf/publicaciones/Cortein

Reglas Mínimas de las Naciones Unidas para la Administración de justicia de Menores. Resolución 40/33 de 28 de noviembre de 1985. http://www.cndh.org.mx.armonlegu $/$ archivos

Directrices de las Naciones Unidas para la Prevención de la Delincuencia Juvenil (Reglas de RIAD), Adoptadas por la Asamblea General de la ONU, en su resolución 45/112 de 1990. $h t t p: / / w w w . i i n . o e a . o r g / c a d-R I A D . p d f$ 